\title{
SHIELDING AREA OPTIMIZATION UNDER THE SOLUTION OF INTERCONNECT CROSSTALK ${ }^{1}$
}

\author{
Xin Zhao, Yici Cai, Qiang Zhou, Xianlong Hong \\ Dept. of CST, Tsinghua Univ. \\ Beijing 100084, P.R.China \\ Tel: +86-10-62785564 \\ Fax: +85-10-62781489 \\ e-mail: plantree99@mails.tsinghua.edu.cn
}

\author{
Lei He, Jinjun Xiong \\ Dept. of EE, UC, Los Angeles \\ Los Angeles, CA 90095-1594, USA \\ Tel: +1-310-206-2037 \\ Fax: +1-310-206-4685 \\ e-mail:lhe@ee.ucla.edu
}

\begin{abstract}
As the technology advances into deep sub-micron era, crosstalk reduction is of paramount importance for signal integrity. Simultaneous shield insertion and net ordering (SINO) has been shown to be effective to reduce both capacitive and inductive coupling. Although shield insertion could reduce crosstalk efficiently, a large scale of unnecessary shields will bring the shielding area problem which is also critical for an efficient SINO algorithm. In this paper, we propose three novel algorithms using fewer shields to solve SINO problem: namely, net coloring (NC), efficient middle shield insertion (EMSI) and NC+EMSI. Compared to the corresponding algorithms in previous work [1], our algorithms can reduce shielding area largely with short runtime.
\end{abstract}

\section{INTRODUCTION}

It has been shown for years that interconnect crosstalk and delay have become bottle necks in determining circuit performance. Even though most current research on interconnect synthesis uses the RC model [2, 3, 4, 5, 6], it is evident that the RLC model becomes more appropriate as the on-chip inductive effect gains increasing prominence in gigahertz designs [7]. As the inductive has long-range effect in the sense that the mutual inductance between non-adjacent nets cannot be ignored when compared to the self inductance [1]. Therefore, shield insertion is needed to reduce inductive noise. However, as shielding area is directly decided by the number of shields, extra shields will waste the shielding area. It is of paramount important to find an efficient way to solve crosstalk problem. The more efficiently shields minimize the crosstalk, the fewer shields need to insert, and the shielding area could be much more optimized. Furthermore, as the increase of IC scale and the number of wires, the short routing resource and the routing congestion become more serious. It is crucial to develop algorithms to reduce the shielding area considering crosstalk at the same time.

Several previous studies have considered interconnect optimization under the RLC model. Assuming that current will return from the nearest shield, the loop inductance model is used in $[1,8,9]$. A table-based partial inductance model is adopted without pre-assuming any current return path $[10,11,12]$; additionally, a coupling inductance screening rule [9] is employed to decide the scope of the current return path. Also, a formula-based $\mathrm{K}_{\mathrm{eff}}$ model is proposed in [8] as the figures of merit for inductive coupling. Although, the assumption of $\mathrm{K}_{\mathrm{eff}}$ model is less intuitive to the designer [7], it is easy to compute and keeps a high fidelity versus the SPICE-computed RLC noise voltage for SINO solutions [8]

Based on $\mathrm{K}_{\mathrm{eff}}$ model [8], Prof He provided greedy shield insertion, graph coloring and simulated annealing SINO algorithms to solve SINO/NB problem [1]. As overabundance shields are inserted in these algorithms, there is still much space for reducing the number of shields to economize the routing resources. In order to optimize the shielding area, in this paper, we develop three algorithms which are extremely efficient to reduce the shielding area while satisfying the crosstalk constraints. First, NC algorithms is proposed to color nets so that no sensitive wires adjacent to each other. Then, considering inserting shield in the key position, the EMSI algorithm is provided to reduce the inductive coupling. Finally, combining $\mathrm{NC}$ and EMSI algorithms into NC+EMSI algorithm, noisebounded problems could be solved using fewer shields.

The remainder of this paper is organized as follows: Section 2 briefly introduces the SINO problem and mostly analyzes algorithms provided in this paper. Section 3 presents three SINO algorithms as Net Coloring algorithm, Efficient Middle Shield Insertion algorithm and NC+EMSI algorithm separately. Section 4 compares experimental results obtained by different algorithms to the existing algorithms [1]. Section 5 concludes the paper.

\subsection{Preliminaries}

\section{PROBLEM FORMULATIONS}

In this paper we consider coplanar interconnect structures [8] with inductive $\mathrm{K}_{\mathrm{eff}}$ model [8]. Shielding area is directly decided by the number of shields with the same width as signal wires (denoted as s-wires). And the group of wires sandwiched between adjacent shields is called a block. Two net $s_{1}$ and $s_{2}$ are defined to be sensitive to each other if a switching signal on $s_{1}$ will cause $s_{2}$ to malfunction or vice-versa [1]. A sensitivity matrix $\mathrm{S}$ is used to represent the sensitivity of wires. In the rest of paper, formula-based $\mathrm{K}_{\text {eff }}$ model [8] is used to compute the inductive coupling coefficient $\mathrm{K}_{\mathrm{ij}}$ between two wires.

\footnotetext{
1 This paper is supported by NSFC(60176016) and High-Tech Research \& Development (863) Program Of China 2002AAIZ1460 and Specialized Research Fund for the Doctoral Program of Higher Education:SRFDP-20020003008
} 


\subsection{Optimal SINO Problems}

Considering capacitive and inductive coupling, SINO/NF and SINO/NB problems are defined in [1] to solve crosstalk minimization. For a given placement $\mathrm{P}$, find a new placement $\mathrm{P}$ ' by simultaneous shield insertion and net re-ordering such that the total area of $\mathrm{P}^{\prime}$ is minimal and $\mathrm{P}^{\prime}$ is noise free. This defines SION/NF problem. In the requirement that $\mathrm{P}^{\prime}$ is capacitive noise free and the inductive coupling $\mathrm{K}_{\mathrm{i}}$ satisfied $\mathrm{K}_{\mathrm{i}} \leq \mathrm{K}_{\text {thresh }}$ where $\mathrm{K}_{\text {thresh }}$ is a uniform thresh value, this forms SINO/NB problem.

\subsection{Problem Analysis}

Shields are used to reduce noise, while overabundance shields may waste the shielding area which is directly decided by the number of shields. Although, existing SINO algorithms [1] reduce both capacitive and inductive noise, there are still lots shields unnecessary. As greedy shield insertion operates [1] inefficiently not aiming at the key position influencing coupling most, we do lots of experiments to find the wires with max $\mathrm{k}$ value.

The coplanar interconnect structures containing 8, 16, 32, 64 s-wires with no shield are used to find the position of wires whose $\mathrm{k}$ value is the max in the block. We iterate the following sensitivity rates: $40 \%, 50 \%$ and $60 \%$ and run each condition under 50 random sensitivities. Table 1 shows the average position of wires having the max $\mathrm{k}$ value.

\begin{tabular}{|c|c|c|c|c|}
\hline sensitivity rate & 8 wires & 16 wires & 32 wires & 64 wires \\
\hline $40 \%$ & 3.10 & 6.05 & 15.42 & 31.24 \\
\hline $50 \%$ & 3.15 & 7.15 & 15.58 & 31.78 \\
\hline $60 \%$ & 3.10 & 7.45 & 15.60 & 31.68 \\
\hline
\end{tabular}

Table 1. Average net position having the max value of $\mathrm{k}$

The experimental results containing $64 \mathrm{~s}$-wires under each $60 \%, 50 \%, 40 \%$ random sensitivities are provided in Figure 1. Here, $\mathrm{x}$-axis indicates the experimental serial number of the 50 random sensitivities, and the $y$-axis indicates the wire order number from 0 to SIZE-1 (where SIZE is the number of wires).

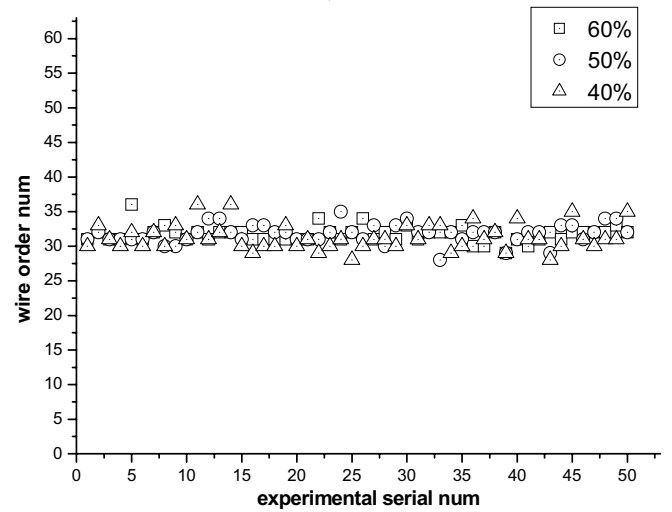

Fig. 1. The wire position of max k value (64 s-wires)

From the Figure 1, we find that $\mathrm{k} \_$max wire always appear from 30th to $32 \mathrm{nd}$ wire. So, the wire having the $\max \mathrm{k}$ value mostly appears in the middle of the block, and by shielding these wires the $\mathrm{k}$ value in the block can be reduced efficiently. Finding this efficient way to reduce noise, fewer shields are needed and the shielding area can be optimized. The efficient middle shield insertion algorithm (EMSI algorithm) is developed based on this discussion.

\section{ALGORITHM FOR OPTIMAL SINO PROBLEMS}

In shielding area optimization, capacitive noise free is defined if wire $\mathrm{s}_{\mathrm{i}}$ is not adjacent to any other wires sensitive to it, and the inductive noise free is defined if sensitive wires do not share a block. We first introduce a Net Coloring algorithm (NC algorithm) to solve noise-free problem, which makes no sensitive wires adjacent to each other. As the SINO/NF problem is overconstrained and may lead to over-designed solutions not according to realistic design constraints, which may need a large number of shields. Also, the EMSI algorithm depends on the initial placement. So we combined NC and EMSI algorithms into $\mathrm{NC}+\mathrm{EMSI}$ algorithm to solve SINO/NB problem. The number of shields can be reduced by first running net coloring algorithm to reorder nets so that no sensitive nets are adjacent to each other, then invoking the EMSI algorithm could reduce inductive coupling noise using shield as few as possible.

\subsection{Net Coloring Algorithm}

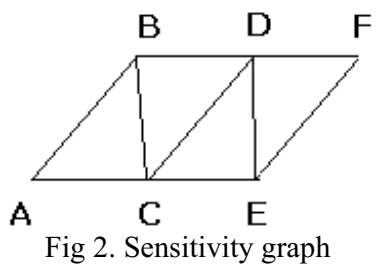

The sensitivity of all wires could also be represented by graph shown in Figure 2, which is constructed as that a node corresponds to a wire and an edge exists if and only if the correspondent wires are sensitive to one another. By coloring the sensitivity graph, wires having the same color are not sensitive to each other.

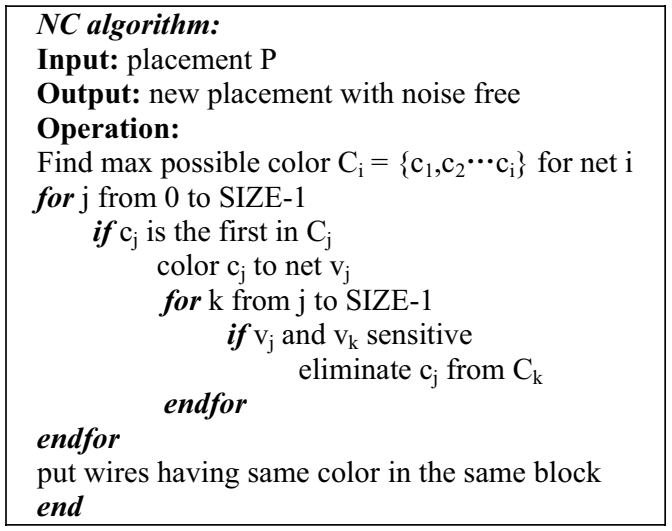

Fig. 3. Net Coloring SINO/NF Algorithm

In Figure 3, we present the net coloring based SINO algorithm to solve noise-free problem. It attempts to color all swires sequentially so that wires in the same block have a single color. Assuming that the set of colors is numbered by 
$\left\{c_{1}, c_{2} \ldots c_{i}\right\}$, where $c_{i}$ represents the $i^{\text {th }}$ color. SINO/NC algorithm works in the following way: First, for each wire $\mathrm{i}$ enumerate the max available set of color $\mathrm{C}_{\mathrm{i}}$, where $\mathrm{C}_{\mathrm{i}}=\left\{\mathrm{c}_{1}, \mathrm{c}_{2}, \ldots \mathrm{c}_{\mathrm{i}}\right\}$. Then color the wires sequentially. Considering each wire, the first color in the set $\mathrm{C}_{\mathrm{i}}$ of wire $\mathrm{i}$ is the final color to wire i. Meanwhile, in order to satisfy no wire sensitive to each other be colorred the same, we delete the color from the set of other pending wires which are sensitive to the wire i considered. Finally, put the wires having the same color in the same block, so that wires in the same block are not sensitive to each other.

\subsection{Efficient Middle Shield Insertion Algorithm}

Efficient middle shield insertion algorithm is mainly about how to insert the shield to solve SINO/NB problem. To optimize the shielding area, we should use as fewer shields as possible to reduce the inductive coupling noise.

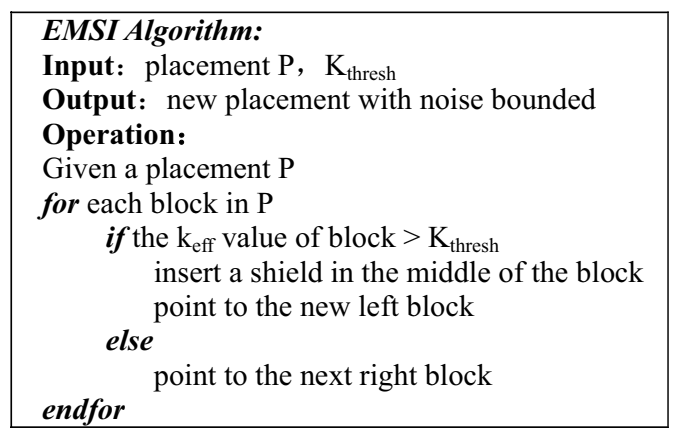

Fig. 4. Efficient Middle Shield Insertion SINO/NB Algorithm

As discussed in section 2, the wire having the max $\mathrm{k}$ value mostly appears in the middle of the block, and by shielding these wires inductive coupling in the block can be reduced efficiently. In Figure 4, we present the efficient middle shield insertion algorithm for SINO problem (SINO/EMSI algorithm). This algorithm is a recursive procedure. Run through all the blocks in the given placement; calculate the max $\mathrm{k}$ value of the block. After inserting a shield in the middle of one block, the algorithm recursively checks the $\mathrm{K}_{\text {eff }}$ value of the two newly-generated blocks. If any of these is still greater than $\mathrm{K}_{\text {thresh }}$, no matter whose $\mathrm{k}$ value is the max, an additional middle shield is inserted in the new block.

\subsection{NC+EMSI Algorithm}

As discussed in section 2, we combined NC and EMSI algorithms into $\mathrm{NC}+\mathrm{EMSI}$ algorithm to solve noise-bounded problem. Net coloring operation is first used to eliminate capacitive noise. While, different from the $\mathrm{NC}$ algorithm which inserts shields between every pair of wires in different colors, net coloring before EMSI algorithm only inserts shields between the adjacent sensitive wires not having the same color. Then efficient middle shield insertion is operated to reduce inductive noise using as fewer shields as possible.

\section{EXPERIMENTAL RESULTS}

In this section, we apply these three algorithms on a large number of examples. And the results obtained by these algorithms are compared to the existing algorithms [1].
We have implemented all algorithms in the $\mathrm{C}++$ programming language on a SUN E450. And we use the coplanar interconnect structures containing 32 and $64 \mathrm{~s}$-wires to determine the performance of the algorithms for different combinations of $\mathrm{K}_{\text {thresh }}$ and uniform sensitivity rate. We use four different $\mathrm{K}_{\text {thresh }}$ values as $0.5,1.0,1.5,2.0$ respectively, here $\mathrm{K}_{\text {thresh }}=0.5$ represents the total inductive coupling coefficient for each net is less than 0.5 . And the sensitivity rate is changed from $40 \%, 50 \%$ to $60 \%$. That the sensitivity rate is $40 \%$ represents each net is sensitive to $40 \%$ of all nets, and these sensitive nets are selected randomly. For each combination of $\mathrm{K}_{\text {thresh }}$ value and sensitivity rate, we present the resulting number of shielding wires for different algorithms. We run each algorithm on the same initial placement for twenty different random sensitivities. And the average of these twenty runs is shown in italic style in table 2 .

\begin{tabular}{|c|c|c|c|c|c|c|c|}
\hline \multirow{2}{*}{$\begin{array}{l}\text { number } \\
\text { of net }\end{array}$} & Kthresh & $\mathrm{GC}[1]$ & $N C$ & $\mathrm{SI}[1]$ & EMSI & $\mathrm{NO}+\mathrm{SI}[1]$ & $N C+E M S I$ \\
\hline & \multicolumn{7}{|c|}{ Net Sensitivity Rate : $40 \%$} \\
\hline \multirow{14}{*}{32} & 0.5 & \multirow[t]{4}{*}{8.50} & \multirow[t]{4}{*}{ 3. 94} & 16.70 & 13. 94 & 6.30 & 6.01 \\
\hline & 1.0 & & & 15.90 & 12.46 & 5.30 & 4.72 \\
\hline & 1.5 & & & 15.40 & 8.68 & 4.40 & 3.99 \\
\hline & 2.0 & & & 13.80 & 6.56 & 4.00 & 3.44 \\
\hline & \multicolumn{7}{|c|}{ Net Sensitivity Rate : $50 \%$} \\
\hline & 0.5 & \multirow[t]{4}{*}{11.00} & \multirow[t]{4}{*}{ 4. 72} & 18.90 & 13.96 & 8.40 & 6.53 \\
\hline & 1.0 & & & 18.40 & 12.46 & 5.70 & 4.97 \\
\hline & 1.5 & & & 18.00 & 8.14 & 4.80 & 4.60 \\
\hline & 2.0 & & & 17.50 & 6.44 & 4.40 & 3.87 \\
\hline & \multicolumn{7}{|c|}{ Net Sensitivity Rate $: 60 \%$} \\
\hline & 0.5 & \multirow[t]{4}{*}{12.00} & \multirow[t]{4}{*}{5.02} & 22.80 & 14.08 & 6.60 & 6.72 \\
\hline & 1.0 & & & 22.10 & 12.36 & 6.00 & 5.30 \\
\hline & 1.5 & & & 22.00 & 8.22 & 5.20 & 4.60 \\
\hline & 2.0 & & & 21.50 & 6.72 & 4.50 & 3.91 \\
\hline \multirow{15}{*}{64} & & & Net $S$ & itivity & ate $: 40$ & & \\
\hline & 0.5 & \multirow[t]{4}{*}{15.60} & \multirow[t]{4}{*}{3.92} & 30.10 & 28.92 & 11.30 & 7.76 \\
\hline & 1.0 & & & 28.50 & 25.96 & 9.20 & 6.88 \\
\hline & 1.5 & & & 26.20 & 18.32 & 7.50 & 5.82 \\
\hline & 2.0 & & & 23.50 & 14.00 & 6.80 & 5.30 \\
\hline & \multicolumn{7}{|c|}{ Net Sensitivity Rate : $50 \%$} \\
\hline & 0.5 & \multirow[t]{4}{*}{ 19. 30} & \multirow[t]{4}{*}{ 4. 78} & 32.10 & 29.12 & 14.30 & 8.17 \\
\hline & 1.0 & & & 31.30 & 25.88 & 9.80 & 7.29 \\
\hline & 1.5 & & & 30.60 & 17.52 & 8.60 & 6.55 \\
\hline & 2.0 & & & 31.50 & 13.96 & 7.90 & 5.71 \\
\hline & \multicolumn{7}{|c|}{ Net Sensitivity Rate $: 60 \%$} \\
\hline & 0.5 & \multirow[t]{4}{*}{21.60} & \multirow[t]{4}{*}{5.11} & 39.70 & 28.72 & 12.80 & 8.21 \\
\hline & 1.0 & & & 39.20 & 25.68 & 11.40 & 7.45 \\
\hline & 1.5 & & & 38.00 & 17.36 & 9.40 & 6.73 \\
\hline & 2.0 & & & 36.80 & 14.40 & 8.10 & 5.85 \\
\hline
\end{tabular}

Table 2. Number of shields inserted by SINO algorithms

For each combination of $\mathrm{k}$ and sensitivity rate, we compare our algorithms investigated above to the existing algorithms [1], the EMSI algorithm is compared to the greedy shield insertion algorithm [1], and the NC+EMSI algorithm is compared to the $\mathrm{NO}+\mathrm{SI}$ algorithm [1], respectively here.

We can see from table 2, the greedy shield insertion algorithm is significantly worse than the EMSI algorithm. For example, in 32 wires with sensitivity rate $(60 \%)$ and $\mathrm{K}_{\text {thresh }}$ changes from $0.5,1.0,1.5$ to 2.0 , EMSI algorithm reduces $27.25 \%, 30.44 \%, 43.06 \%, 46.19 \%$ shields respectively compared to SI algorithm [1]. Furthermore, as we expected, the EMSI algorithm has a higher efficiency to reduce the inductive coupling noise, along with the increase of $\mathrm{K}_{\text {thresh }}$, the number of shields by EMSI algorithm reduces obviously, while the result of SI algorithm [1] reduces insignificantly. See 64 s-wires, sensitivity rate $=50 \%$, when $\mathrm{K}_{\text {thresh }}$ increase from $0.5,1.0,1.5$ to 2.0 separately, the number of shields in EMSI algorithm to the given size (64 s-wires) decreases from $45.50 \%, 40.44 \%, 27.38 \%$ to $21.81 \%$ respectively with $23.69 \%$ reduction, but the results of 
SI algorithm are $50.16 \%, 48.91 \%, 47.81 \%, 49.22 \%$ with at most $2.35 \%$ reduction. So, middle shield insertion in EMSI algorithm may efficiently reduce the inductive coupling noise, and the shielding area reduces significantly.

Compared to the graph coloring algorithm [1], the net coloring algorithm also needs $18.25 \%, 22.69 \%$ and $25.77 \%$ less shields separately than SINO/GC algorithm [1] in 64 s-wires with sensitivity rate from $40 \%, 50 \%$ to $60 \%$.

Considering separated net ordering and shield insertion operation [1], NC+EMSI algorithm not only follows the requirement of net ordering operation about eliminate the capacitive coupling noise, but also gathers wires not sensitive to each other into the same block by the greatest extent, which is helpful to the following shield insertion operation to reduce the inductive coupling noise. From the experimental results, $\mathrm{NC}+\mathrm{EMSI}$ algorithm also shows a good performance. Take $64 \mathrm{~s}-$ wires with sensitivity rate $60 \%$ and $\mathrm{K}_{\text {thresh }}$ value increases from $0.5,1.0,1.5$ to 2.0 as example, the $\mathrm{NO}+\mathrm{SI}$ algorithm [1] needs $20.00 \%, 17.81 \%, 14.69 \%, 12.66 \%$ shields, while relatively, the $\mathrm{NC}+\mathrm{EMSI}$ algorithm needs $12.83 \%, 11.64 \%, 10.52 \%, 9.14 \%$ shields to the given size 64 , which means $7.17 \%, 6.17 \%, 4.17 \%$, $3.52 \%$ less shielding area than the NO+SI algorithm[1].

\begin{tabular}{|r|l|l|l|}
\hline & EMSI & NC & NC+EMSI \\
\hline Runtime & $0.013 \mathrm{sec}$ & $0.00 \mathrm{sec}$ & $0.004 \mathrm{sec}$ \\
\hline Table 3. & \multicolumn{2}{|c|}{$\begin{array}{c}\text { Approximate run times for SINO algorithms } \\
\text { with sensitivity rate } 60 \%\end{array}$}
\end{tabular}

Finally, the runtime is showed in Table 3, where the times are for a single interconnect structures with a single run. SUN E450 is used to collect running time. From this table, all algorithms finished the examples in a few seconds. Therefore, large scale interconnect structures can be solved fast by algorithms we have proposed.

\section{CONCLUSION AND DISCUSSIONS}

As more and more high performance microprocessors and system-on-chip (SoC) operates in $\mathrm{GHz}^{+}$scale, RLC crosstalk gains growing importance for signal integrity. In this paper, we have developed three efficient algorithms to solve the shielding area optimization. Extensive experiment results have shown that compared to the previous corresponding algorithms in [1] the proposed algorithms use less runtime while reduce the shielding area efficiently. SINO is a region based technique to reduce RLC crosstalk. Performing SINO within each region separately may introduce many dog-legs across regions. For full-chip routing optimization, we want to reduce the number of dog-legs as they deteriorate signal integrity [6]. We plan to study the row-based SINO problem with consideration of both shielding area and dog-leg minimization in the future, and develop efficient algorithms correspondingly.

\section{REFERENCES}

[1] L. He and K. M. Lepak, "Simultaneous shield insertion and net ordering for capacitive and inductive coupling minimization", in ISPD, pp. 55-60, 2000

[2] Gao, T. and Liu, C. L. "Minimum Crosstalk Channel Routing”, in ICCAD, pp.692-696, 1993.

[3] Gao, T. and Liu, C. L. "Minimum crosstalk switchbox routing", in ICCAD, pp. 610-615, 1994.

[4] Yim, J. S. and Kyung, C. M. "Reducing Cross-Coupling amongn Interconnect Wires in Deep-Submicron Datapath Design”, in DAC, pp.485-189, June, 1999.

[5] Xue, T. and Kuh, E. S. "Post global routing crosstalk synthesis", in IEEE Trans. CAD-16, pp.1418-1430, 1997.

[6] Chang, C.-C. and Cong, J. "Pseudo pin assignment with crosstalk noise control", in ISPD, pp. 41-47, April, 2000

[7] K. M. Lepak, I. Luwandi, and L. He, "Shield insertion and net ordering under explicit RLC noise constraint", in DAC, pp.199-202, June, 2001

[8] L. He and M. Xu, "Modeling and Layout Optimization for On-chip Inductive Coupling", U. of Wisconsin at Madison, Technical Report ECE-00-1

[9] S. Lin, N. Chang, and O. S. Nakagawa, "Quick on-chip selfand mutual-inductance screen", in International symposium on Quality of Electronic Design, pp.513-520, March, 2000.

[10] A. Ruehli, "Equivalent circuit models for three-dimensional multiconductor systems", IEEE Trans. On MIT, pp.216-221 , 1974.

[11] L. He, N. Chang, S. Lin, and O. S. Nakagawa, "An Efficient Inductance Modeling for On-Chip Interconnects", in IEEE CICC, pp.457-460, May, 1999.

[12] J. D. Ma and L. He, "Formulae and Applications of Interconnect Estimation Considering Shielding Insertion and Net Ordering”, IEEE/ACM ICCAD, pp.327-332, November 2001. 